\title{
Acute necrotic myelitis and perivenous encephalomyelitis associated with hypertension and renal infection
}

\author{
A. A. MILLER AND F. RAMSDEN \\ From the Group Laboratory, Preston Royal Infirmary
}

SYNOPSIS The case reported showed acute necrotic myelopathy and acute perivenous encephalomyelitis against a background of renal infection, malignant hypertension, and a history of injury. Death from renal disease and pneumonia halted the neurological pathology in the acute stage, thus allowing a more detailed histological examination than is possible in the later stages. The pathogenesis of acute necrotic myelopathy, together with the effects of oedema and vascular compression in the cord, are discussed briefly.

The case reported here shows the rare histological association of 'acute necrotic myelopathy' (Hoffman, 1955) with acute disseminated encephalomyelitis of the perivenous type. Few of the similar lesions previously reported have shown oedema and softening of the spinal cord (de Vries, 1960). An unusual feature was the association of hypertension and acute necrotic myelopathy.

\section{CLINICAL HISTORY}

A woman, aged 20 years, and giving a history of chronic nephritis with frequent epistaxis, was treated in hospital for both these conditions, together with left ventricular failure. Her blood pressure fluctuated between 170/110 and $210 / 150 \mathrm{~mm}$. Hg. Treatment included antibiotics, digoxin, diuretics, blood transfusion, anabolic drugs, and a low-protein diet: hypotensive drugs were not used.

About five months later she knocked her back at a local fairground but did not complain afterwards. Eleven days later she felt weakness in both legs, was unable to walk, and fell to the floor.

On admission to hospital there was flaccid paraplegia of both legs, with loss of sensation below the sixth thoracic level, followed by urine retention and neck stiffness. A myelogram on the third day did not show evidence of spinal block. The patient was transferred to a neurosurgical unit (Mr. G. K. Tutton) on the fourth day: the sensory level had now reached the second thoracic segment; the patient was fully conscious with pain and stiffness in the back of the neck and pins and needles' in both arms. There was flaccid paraplegia of both legs with absence of tendon and plantar reflexes. Abdominal reflexes were absent and there was urinary

Received for publication 21 April 1967. incontinence. Speech was not slurred; cranial nerve functions were normal; the optic discs were blurred. Weakness of the arms was not demonstrated. Temperature and respiration rate were normal; the pulse rate was 100 per minute and the blood pressure $210 / 150 \mathrm{~mm}$. Hg.

By the sixth day there was marked weakness in the upper limbs and eventually loss of power with absent reflexes: this was followed by chest symptoms, with marked cough, and bilateral diaphragmatic weakness. The patient showed increasing mental confusion, became drowsy, comatose, and eventually died on the tenth day of neurological illness. Steroids had not been used as part of her treatment.

Laboratory investigations included examination of cerebrospinal fluid: this contained $471 \mathrm{mg}$. per $100 \mathrm{ml}$. protein with $690 \mathrm{mg}$. per $100 \mathrm{ml}$. chloride: the white cell count was 101 per c.mm. (90\% polymorphs, $10 \%$ lymphocytes).

During the period in hospital there was normocytic, normochromic anaemia (haemoglobin $5.6 \mathrm{~g}$. per $100 \mathrm{ml}$.) and the blood urea was consistently high (500 mg. per $100 \mathrm{ml}$.), both these findings associated with chronic nephritis.

On first admission the white cell count was normal but marked lymphopenia developed. The urine contained protein, red blood cells, and pus: cultures gave a profuse growth of a coliform organism.

\section{NECROPSY}

The examination was performed about 18 hours after death, the causes of death being uraemia, due to malignant nephrosclerosis, and bilateral suppurative bronchopneumonia.

The heart $(500 \mathrm{~g}$.) showed hypertrophy of the left 
ventricular wall and interventricular septum, the substance being congested but not fibrosed; valves were competent; coronary arteries were patent with slight atheromatous degeneration.

Endocrine glands appeared normal apart from an enlarged, congested pituitary gland. Enlarged lymph nodes were present throughout the body; the spleen (180 g.) was congested.

MACROSCOPICAL EXAMINATION OF THE CENTRAL NERVOUS SYSTEM The brain was of normal size and without ventricular distension, the leptomeninges congested with some haemorrhage. On coronal section there was congestion throughout the cerebral hemispheres and in the floor of the fourth ventricle with smaller zones lower in the medulla. Areas of softening were not found.

The spinal cord appeared normal in its upper part, but, from C 5 down to the lumbar segments, was swollen and soft with engorged superficial blood vessels. Section showed haemorrhagic areas from C 6 down to $L 3$ with obvious necrosis from the mid-thoracic to upper lumbar parts.

MICROSCOPICAL EXAMINATION OF THE CENTRAL NERVOUS SYSTEM In the white matter of the right occipital lobe, the isthmus of the temporal lobe, the cerebral cortex, basal ganglia and the pons there were a few small perivenous foci of demyelination which had occasionally coalesced to form larger areas. The cerebellar cortex appeared normal: there were degenerative changes in the nerve cells of the dentate nucleus.

The cranial nerves, including the whole of the optic tract, appeared normal apart from leptomeningeal round-cell infiltration.

There was perivascular round-cell infiltration in the pituitary gland with congestion of intraglandular blood vessels.

In the spinal cord, at the level of C 3, myelin stains showed diffuse pallor of grey and white matter, including the basal parts of the posterior columns. This pallor increased in the lower cervical segments, gradually including frank demyelination in posterior columns and eventually in the lateral white columns: small perivenous foci had joined to form larger areas.

The changes became progressively more marked down the lower cervical and thoracic parts until, at $\mathrm{T} 11$ and 12, the whole cross-sectional area of the cord was almost completely demyelinated and necrotic (Fig. 1). In the lumbar and sacral regions the intensity of the pathological process decreased, first the anterior columns and eventually (L 4 and 5) the posterior columns being spared.

Damage to neurones was obvious at $\mathrm{C} 5$, became more marked in the thoracic region, where ganglion

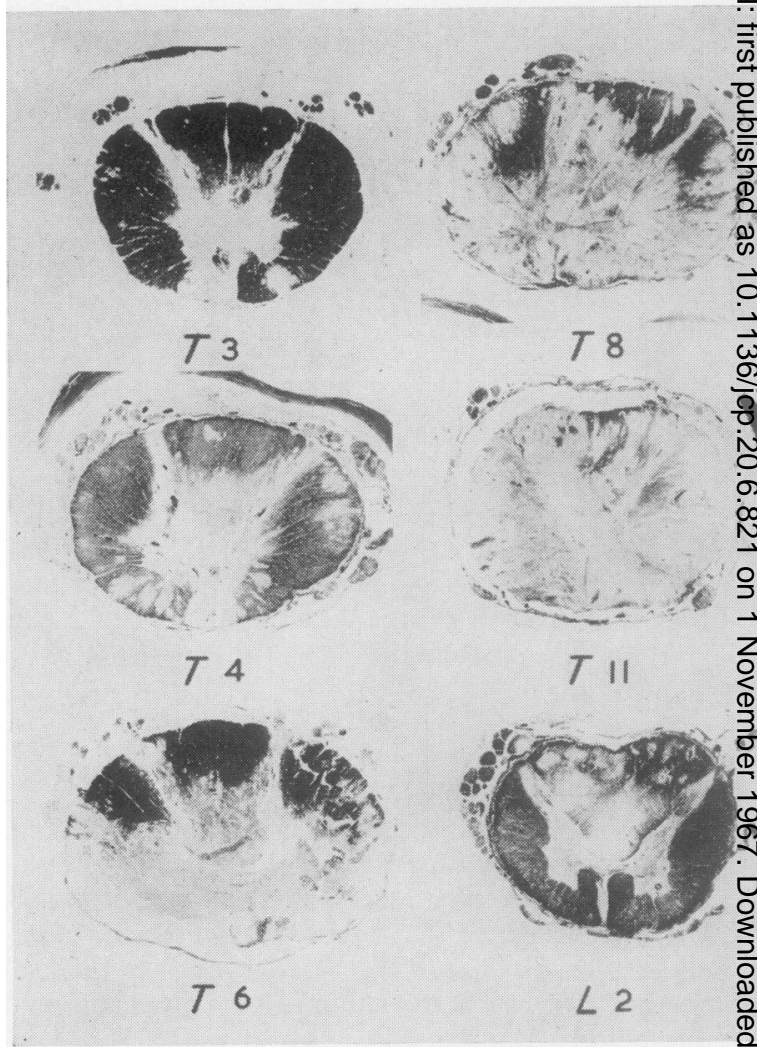

FIG. 1. Sections from the thoracic and lumbar cord showing varying degrees of demyelination and necrosis. Weigert-Pal. $\times 3$.

cells were almost absent, but there was progressively less damage in the lumbar and sacral segments (Fig. 2).

Excepting in the thoracic region, microglial proliferation was not seen: astrocytic increase was not obvious. Fat granule cells were associated with demyelinated areas and their numbers varied with the severity of the process.

Intramedullary spinal blood vessels showed mural hyalinization and cuffing with round cells: perivascular haemorrhage was seen in some upper thoracic segments. Blood vessels in the mid-thoracic region were surrounded by pools of oedema; many were flattened and bloodless (Fig. 3). Extraspinal arteries were less affected: slight endothelial proliferation of the anterior spinal artery was present as part of a mild atheromatous change. Thrombosis and gross atherosclerosis were not evident in any of the blood vessels related to the cord and the lesions could not be explained on a purely vascular basis.

In the thoracic region the proximal parts of the $\stackrel{\triangle}{\triangle}$ posterior nerve roots were almost completely 

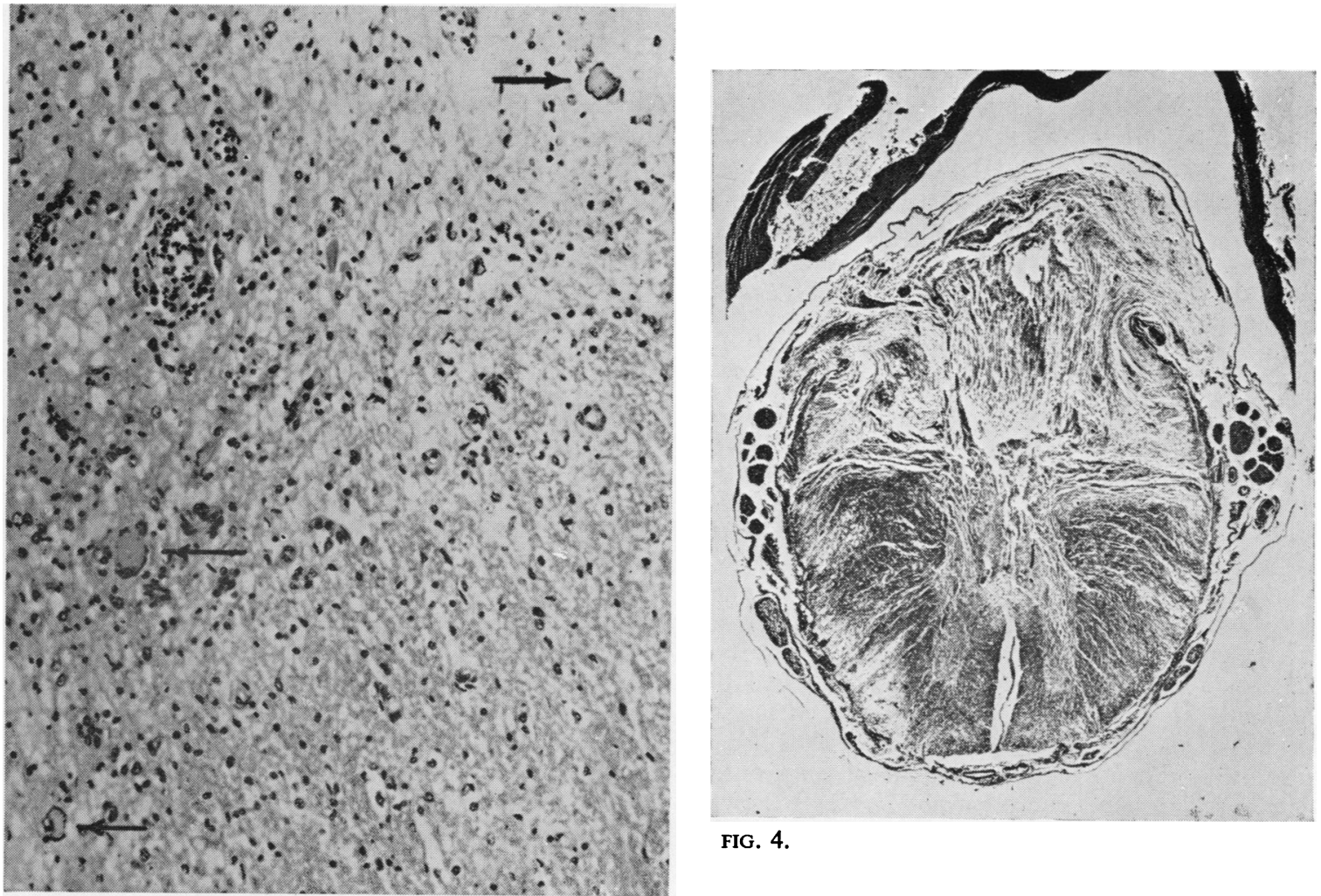

FIG. 4.

FIG. 2.

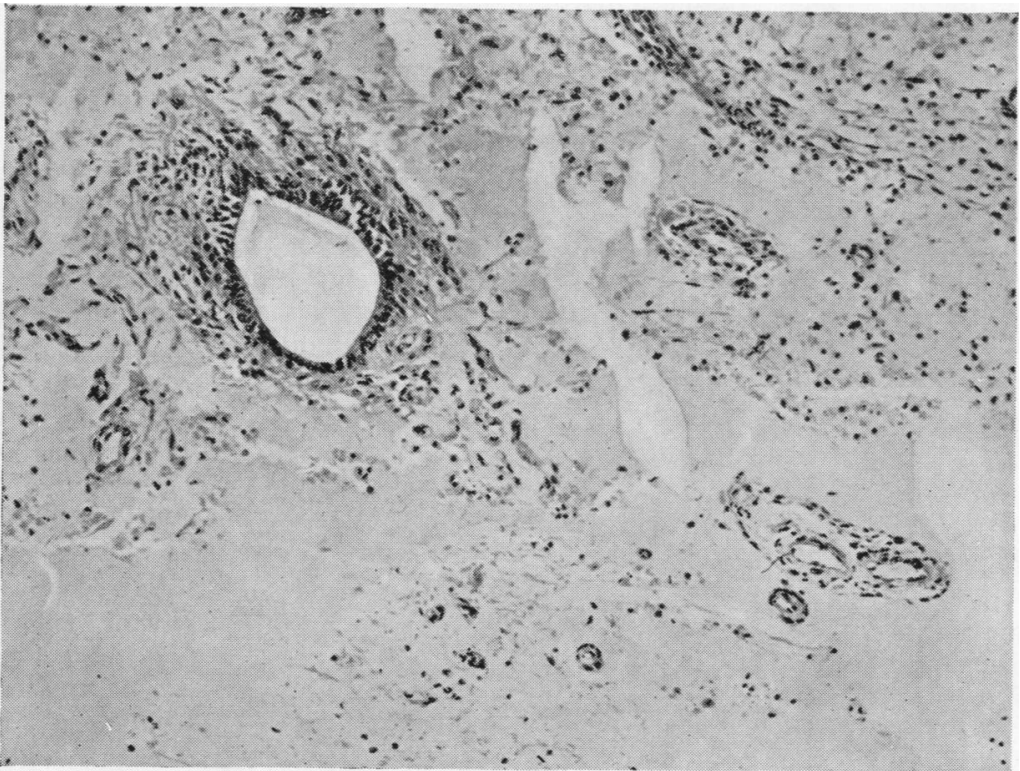

FIG. 2. C.5. Part of the anterior horn is demyelinated and oedematous: the few surviving ganglion cells appear swollen and chromotalytic. Haematoxylin and eosin. $\times 140$.

FIG. 3. T.9. The central grey commissure is largely replaced by pools of oedema in which a few islands of blood vessels are present. Haematoxylin and eosin. $\times 100$.

FIG. 4. L.1. Perforation of dorsal and lateral pia with extrusion of cord substance into the subarachnoid space. Phosphotungstic acid-haematoxylin. $\times 6$.

FIG. 3 . 
demyelinated. There was herniation of cord substance into dural sleeves of anterior and posterior nerve roots in the lower thoracic and upper lumbar segments. Perforations of dorsal and lateral pia (L 1) had allowed prolapse of cord substance into the subarachnoid space (Fig. 4).

Histological examination of tissues outside the central nervous system did not show evidence of endocrine abnormality. Other sections confirmed the macroscopic findings in lungs, heart, kidneys, and spleen.

\section{DISCUSSION}

Reported cases of acute necrotic myelopathy of the spinal cord present a remarkably similar distribution pattern. The necrosis is mainly in the middle levels, with a predilection for the base of the posterior columns; the gross lesion usually assumes a spindle shape in longitudinal section (Hoffman and Norman, 1964; Hughes, 1966). The factors determining this shape and the mechanism of necrosis are not certain. Hoffman (1955) and Hewer (1964) suggested that oedema of the cord created pressure within the inelastic pia and that the rising pressure led to necrosis: the cause of the pressure has been variously cited as extensive demyelination, space-occupying lesions, such as abscesses within the cord, and vascular insufficiency following trauma. Increased pressure, however caused, could lead to compression of blood vessels and subsequent ischaemic necrosis. Many suggest that necrotic myelopathy is fundamentally a vascular change, the demyelination and necrosis being secondary, but there seems sufficient evidence to suggest that the vascular and cord changes are simultaneous in many cases.

Acute necrotic myelopathy following bacterial infection outside the central nervous system was discussed by Jaffe and Freeman (1943) and Hoffman (1955).

Amongst reports of similar lesions following serum therapy there are two (Riser, Geraud, and Planques, 1937; Miller and Ramsden, 1962) where acute necrotic myelopathy and perivenous encephalomyelitis followed injection of antitetanus serum. Similar reactions have been attributed to a variety of foreign proteins and other allergens, including viruses and bacterial toxins, the presence or absence of primary vascular change depending upon the particular stimulus.

In hypersensitive states the altered permeability of the blood vessel endothelium (Waksman and Adams, 1955) will permit antibodies to reach the cord substance: these antibodies, reacting with fixed tissue antigens, could produce tissue lysis. The myelin sheath could act as a fixed tissue antigen, and, being lipid-rich, would be an ideal substrate for the lysolecithin released following an antigen-antibody reaction, a mechanism similar to that proposed byo Pette and Pette (1963). By such means blood-borne을 materials could participate in perivascular antigen- $\frac{\bar{p}}{\overline{\frac{1}{3}}}$ antibody reactions with resultant demyelination and $\stackrel{\Phi}{\varnothing}$ oedematous swelling, the increased volume producing raised pressure within the inelastic pia. The only solutions to this hydraulic problem are. compression of blood vessels or extrusion of cord $\overrightarrow{\vec{\omega}}$ substance through weaker points in the pia: theo ischaemia resulting from compression of blood vessels would lead to secondary necrosis and oedemain with still further increase in pressure.

The present patient did not give a history of $\dot{\circ}$ treatment with steroids or other potentially toxic ${ }_{-}^{N}$ drugs: virus investigations gave negative results. 을 Urinary tract infection had been present for longperiods and the toxins produced could have initiated $z$ a mechanism similar to that described above; certainly the histological findings were consistent with this.

Published work on acute necrotic myelopathy includes 23 cases without evidence of hyper- $\vec{\mathscr{}}$ tension but in three others (Greenfield and Turner,. 1939; Jaffe and Freemann, 1943; Hoffman, 1955) there was hypertension. In the case reported here the renal changes, being of long duration, could not $\frac{D}{0}$ have followed myelopathy but malignant hypertension resulting in vascular damage to the cord $\stackrel{\circ}{\Phi}$ does seem possible. The measured blood pressures $\vec{F}$ were often not grossly raised but the finding of $\frac{\circ}{3}$ cardiac enlargement $(500 \mathrm{~g}$.) suggests that the higher pressures recorded $(210 / 150 \mathrm{~mm}$. $\mathrm{Hg})$ may have been present for long periods (Dickinson, 1966). The association between hypertension and necrotic? myelopathy is rare and, in this case, a causal relationship appears doubtful.

Jaffe and Freeman (1943) reviewed cases of spinal $\stackrel{8}{\circ}$ necrosis and included two which had followed direct injury to the back. In one of these the findings $\frac{\text { ㄱ }}{7}$ suggest to us that the necrosis was a result of $D$ subsequent infection rather than the initial injury: the other case appears to show a causal relationship $N$ between injury and subsequent necrosis. The review also includes three cases where similar lesions had $\tilde{\circ}$ followed straining of the back, but, in one of these there was also thrombosis of spinal blood vessels. 웅

In our case the interval of 11 days between injury and onset of symptoms makes a direct connexion $\overparen{\Phi}$ appear tenuous: there was no significant symptom $\stackrel{\mathcal{f}}{\rightarrow}$ or complaint of anything suggesting vascular spasm 0 or occlusion during this period.

One case of acute necrotic myelopathy with $\stackrel{\mathbb{Q}}{\stackrel{D}{\circ}}$ acute perivenous encephalomyelitis (Hoffman and $\mathbb{Q}$ Norman, 1964) appears complementary to ours. $A_{\frac{2}{2}}$ 
male, aged 57 years with a long history of chronic chest infection, developed right-sided paralysis followed by extensive damage to the spinal cord and died on the 26th day of neurological illness. Necropsy showed acute necrosis and perivenous encephalomyelitis in the cord, perivascular demyelination in the cerebrum and optic tract, together with oedematous necrosis in the cerebellum. This case showed the terminal phase of the process whereas ours showed the acute phase, death from other causes having halted the process after 10 days.

The association of post-exanthematous encephalomyelitis and acute cord necrosis was reviewed by Hoffman and Norman (1964): Hoffman (1955) also reviewed the literature on acute necrotic myelopathy which was not associated with perivenous encephalomyelitis. Their comments on the literature are relevant to the case reported here.

Consideration of available information in this case and comparison with other reported material suggests that the acute necrotic myelopathy resulted from a hypersensitivity reaction to bacterial toxins: other possible causes, such as hypertension and injury, appear less likely.

We are indebted to Dr. J. T. Hughes for reviewing the spinal cord histology: to Dr. C. F. Dickinson for his comments on hypertensive states; to Mr. G. K. Tutton for access to clinical material; to Messrs. J. H. Wilkinson and $\mathrm{J}$. Urquhart for numerous histological preparations and to Miss P. S. Farnworth for secretarial help.

\section{REFERENCES}

Dickinson, C. F. (1966). Personal communication.

Greenfield, J. G., and Turner, J. W. A. (1939). Brain, 62, 227.

Hoffman, H. L. (1955). Ibid., 78, 377.

, and Norman, R. M. (1964). J. Neurol. Neurosurg. Psychiat., 27, 116.

Hughes, J. T. (1966). Pathology of the Spinal Cord, p. 154, Lloyd-Luke, London

Jaffe, D., and Freeman, W. (1943). Arch. Neurol. Psychiat. (Chic.), 49, 683.

Miller, A. A., and Ramsden, F. (1962). J. clin. Path., 15, 314.

Pette, E., and Pette, H. (1963). J. Neuropath. exp. Neurol., 22, 528.

Riser, Geraud, and Planques, (1937). Rev. Neurol., 67, 455.

de Vries., E. (1960). Postvaccinal perivenous encephalitis. (Folia Psychiatrica, Suppl. no. 5). Elsevier, Amsterdam.

Waksman, B. H., and Adams, R. D. (1955). J. exp. Med., 102, 213. 